\title{
Generalized Weighted Fairness Criterion : Formulation and Application on Prioritized ABR Service
}

\author{
Clarence S. C. Lee, K. F. Cheung, Danny H. K. Tsang \\ Electrical and Electronic Engineering Department \\ The Hong Kong University of Science \& Technology \\ Clear Water Bay, Kowloon, Hong Kong
}

\begin{abstract}
In this paper, a generalized fairness criterion referred to as the Generalized Weighted Fairness Criterion (GWFC) for flow control on $A B R$ service is presented. Within the GWFC framework, a weight is assigned to each ABR connection and bandwidth is allocated to it in proportion to the corresponding weight. The GWFC can generalize fairness sub-criteria for prioritized services, and in particular, subsume the Max-Min criterion as a uni-weight fairness criterion. Two weighted fairness sub-criteria are introduced and their performance on bandwidth allocation will be presented and compared with that of the Max-Min criterion.
\end{abstract}

\section{Introduction}

Fair share allocation on residual bandwidth is the main concern for the design of ABR flow control algorithms. A major drawback of the Max-Min criterion [1,2] which has been adopted by the ATM forum is the beat down problem [3]. This is because every ABR connection takes the smallest bandwidth allocation over all the ones obtained along the traversing links. Hence, every ABR connection is penalized by the number of links it takes.

Another weakness of the Max-Min criterion is its inability to provide prioritized ABR service. With the GWFC, ABR subscribers can pay for a larger amount of bandwidth [4] and the ABR vendors can implement priority strategies in order to achieve certain quality-of-service (QoS) attributes.

In this paper, a generalized fairness criterion referred to as the Generalized Weighted Fairness Criterion (GWFC) is formulated. Within the GWFC framework, a weight is assigned to each ABR connection and bandwidth is allocated to it proportional to the corresponding weight. In particular, the Max-Min criterion is subsumed within the GWFC as an uni-weight fairness criterion. In general, the GWFC can generalize fairness criteria which are required for providing prioritized services.

Based on the GWFC, two sub-criteria are introduced and their performance are demonstrated. The first criterion introduced is the Proportional-to-Link (PL) criterion, with which the weight assigned is proportional to the number of links a connection traverses. As will be shown, the PL criterion is effective in solving the beat down problem. The second proposed criterion is the Minimum-Cell-Rate (MCR) criterion with which the weight assigned is proportional to the MCR of the corresponding connection. Formulation of the MCR criterion is aimed at providing QoS-based prioritized ABR services. As will be shown, ABR subscribers who are willing to subscribe higher MCR values will be allocated with more favorable shares of bandwidth.

\section{Generalized Weighted Fairness Criterion}

A network is assumed to be a directed graph $G=(N, L)$, where $N$ is the set of nodes and $L$ is the set of links in the graph. Connection $p \in P$, where $P$ is the set of $\mathrm{ABR}$ connections in the network, denotes a fixed route of an ABR connection. Let $L_{p}$ denote the sets of links traversed by $p$ and $U_{l}=\left\{p \mid l \in L_{p}\right\}$ represent the set of connections supported by link $l$. The notations $C_{l}$ and $\mu_{l}, l \in L$, denote the capacity and target utilization of link $l$, and $m_{p}$ is the MCR value of connection $p . \quad r_{p}$ is the rate allocation of connection $p$ and $\alpha_{l p}$ is defined as the weight of connection $p$ at link $l$ which represent the priority of the connections. The rate set $R=\left\{r_{p} \mid p \in P\right\}$ consists of the rate allocations of all connections.

In the framework of the GWFC, the weighted fair share of bandwidth, $f(l, p)$, allocated to connection $p$ at link $l$ is proportional to the weight $\alpha_{l p}$ which is assigned to the connection by the network. Thus,

$$
f(l, p)=\left(C_{l} \cdot \mu_{l}-\sum_{p^{\prime} \in U_{l}} m_{p^{\prime}}\right) \cdot w_{l_{p}}+m_{p}
$$




$$
, p \in U_{l}, l \in L
$$

where $w_{l p}$ is defined as

$$
w_{l p}=\frac{\alpha_{l p}}{\sum_{p^{\prime} \in U_{l}} \alpha_{l p^{\prime}}} .
$$

The resultant bandwidth assigned to connection $p$ is the minimum of allocated bandwidth among all the links that $p$ traverses. Accordingly, the weighted fair share for connection $p$ is

$$
g(p)=\min _{l \in L_{p}} f(l, p) .
$$

Next, an algorithm is outlined below for computing the weighted fair rate set $R$. By using (3), it updates each connection and continues to fully utilize remaining bandwidth due to constrained connections. When all connections are constrained at one or more bottleneck links, the link capacities are allocated optimally and the algorithm stops.

In this algorithm, $F_{l}^{j}$ and $P^{j}$ represent the total allocation of link $l$ and the set of unconstrained connections at the beginning of step $j$ respectively. The algorithm with the initial conditions of $j=1$ and $r_{p}^{0}=g(p), p \in P$, is given as follows :

$\begin{aligned} & \text { 1. } F_{l}^{j}:=\sum_{p \in U_{l}} r_{p}^{j-1} \quad, l \in L \\ & \text { 2. } P^{j}:=\left\{p \mid\left(C_{l}-F_{l}^{j}\right)>0, \forall l \in L_{p}, p \in P\right\}\end{aligned}$

3. If $P^{j}$ is empty, then $R=\left\{r_{p}^{j-1} \mid p \in P\right\}$

is formed and the algorithm stops.

4. $r_{p}^{j}:= \begin{cases}r_{p}^{j-1}+\left\{\min _{l \in L_{p}}\left[\left(C_{l}-F_{l}^{j}\right) .\right.\right. & \\ \left.\left.\frac{\sum_{p^{\prime}} \in U_{l} \cap P^{j}}{\alpha_{p_{p}}}\right]\right\} & , p \in P^{j} \\ r_{p}^{j-1} & \text { o.w. }\end{cases}$

5. $j:=j+1$, go to 1 .

\subsection{Max-Min Fairness Criterion}

In the GWFC framework, the Max-Min criterion is a uni-weight criterion with :

$$
\alpha_{l p}=\alpha_{p}^{M M}=1 \quad, p \in P, l \in L .
$$

where residual capacity is divided equally by the number of connections.

\subsection{Proportional-to-Link Criterion}

With this criterion, the weight is proportional to the number of links connection $p$ traverses, which is denoted by $k$ and is written as,

$$
\begin{gathered}
\alpha_{l p}=\alpha_{p}^{P L}=B \cdot\left(1-e^{-\beta \cdot(k-1)}\right)+\alpha_{p}^{P L}(1) \\
, p \in P, l \in L
\end{gathered}
$$

where $\alpha_{p}^{P L}(1)$ represents the priority for connections that traverse only one link and serves as the reference point. In particular, it will be set to one in the following discussion. The exponent value $\beta$ is determined numerically to be 0.7 which is the best value for combating the beat down problem. As shown in Fig.1, weights of connections that traverse more links will be larger to counter the beat down problem. The weight profiles become saturated when they reach the upper bound of the priority, $B$, which is incorporated to prevent domination of link resources by a connection.

\subsection{Minimum-Cell-Rate Criterion}

With this criterion, weight is assigned equal to the MCR value :

$$
\alpha_{l p}=\alpha_{p}^{M C R}=m_{p} \quad, p \in P, l \in L,
$$

where $m_{p}$ is the MCR value of connection $p$. This criterion aims at providing QoS-based prioritized ABR service.

\section{Performance analysis}

In this section, we will compare Max-Min, MCR and PL criteria, with respect to the expected bandwidth allocation for connections that traverse different number of links. In particular, we intend to study analytically the improvement on the beat down problem with the PL criterion and the bandwidth allocation of the priority connections with the MCR criterion.

Consider a connection $p$ that traverses $k$ links. Let $N_{j}$ be a random variable denoting the number of connections sharing the $j$ th link along the path traversed by $p$. To derive the expected bandwidth allocation, the following assumptions are made :

- Traffic load for the network is symmetrical.

- All link capacities are normalized to unity.

- Target utilization of each link is $100 \%$.

- The number of connections sharing the $j$ th link $\left(N_{j}\right)$, $j=1,2 \ldots k$, are independent events.

- Each ABR source can fully utilize the bandwidth allocated.

\subsection{Max-Min Criterion}

For the Max-Min criterion, let $S_{k}=$ $\max \left(N_{1}, N_{2}, \ldots, N_{k}\right)$ be the number of connections at the bottleneck link. The probability that there are $i$ connections at the bottleneck link of $p$ is

$$
P\left(S_{k}=i\right)=\prod_{j=1}^{k} P\left(N_{j} \leq i\right)-\prod_{j=1}^{k} P\left(N_{j} \leq i-1\right) .
$$


Specifically, let $N_{j}, j=1,2, \ldots, k$, be uniformly distributed from one to $M$ (i.e. $N_{j} \leq M$ ), where $M$ denotes the maximum number of connections supported by a link. Equation (8) for $i=1,2 \ldots, M$ can be simplified to

$$
P\left(S_{k}=i\right)=\frac{1}{M^{k}}\left[i^{k}-(i-1)^{k}\right] .
$$

Let the MCR of all the connections be zero. The expected share of bandwidth with the Max-Min criterion can be simplified to

$$
E_{M M}[g(p)]=\sum_{i=1}^{M} P\left(S_{k}=i\right) \cdot \frac{1}{i} .
$$

As shown in Fig.2, the expected bandwidth for the Max-Min fairness criterion decreases monotonically and approaches the limit of minimum share $(1 / M)$ with increasing $k$. Such phenomenon indicates that multi-link connections are penalized with respect to bandwidth allocation.

\subsection{Proportional-to-Link Criterion}

We now proceed to analyze the PL criterion. Specifically, all connections encountered by $p$ at its bottleneck link are assumed to traverse $n$ links, where $n$ is defined as the mean number of links traversed by the connections in the network. Under the symmetric assumption, with reference to (6) and (9), the expected bandwidth allocation is

$$
\begin{aligned}
E_{P L}[g(p)]= & \sum_{i=1}^{M}\left\{P\left(S_{k}=i\right) .\right. \\
& \left.\frac{\alpha^{P L}(k)}{\alpha^{P L}(k)+(i-1) \cdot \alpha^{P L}(n)}\right\} .
\end{aligned}
$$

Results in Fig.3 shows that with the weights in accordance with (6), bandwidth can be allocated to the connections equally and independent of the number of link traversed.

As shown in Fig.4, small values of $B$ are not enough to compensate for the beat down effect while large values of $B$ would cause connections with more links to be favored more than those with fewer links. With an optimum value of $B$, the beat down problem can be eliminated. In Fig.5, when $n$ is large, the chosen $B=8$ is smaller than the optimum value and is not enough to compensate for the beat down problem. With large values of $n$, multi-link connections are given too much priority.

We now introduce the procedures to estimate the optimum value of $B$. Clearly, $B$ should be chosen to minimize the difference in bandwidth allocation for connections that traverse different number of links. Rewrite the expected bandwidth allocation for the PL criterion as $E(k, B)$ and let $K$ be the maximum number of links traversed by a connection. Also assume connection $p$ traversing $k$ link, $k=1,2, \ldots K$, with equal probability. We can then define a variance measure

$$
V(B)=\frac{1}{K} \cdot \sum_{k=1}^{N}[E(k, B)-\bar{E}]^{2},
$$

where $\bar{E}$ is the mean of $E(k, B)$ and is given by

$$
\bar{E}=\frac{1}{K} \cdot \sum_{k=1}^{N} E(k, B) .
$$

The optimum value of $B$ can be found by solving the following minimization problem :

$$
\min _{B \in[0, \infty)} V(B) .
$$

As a result, given $n, M$ and $K$ which can be determined from the network information, the optimum value of $B$ can be found.

\subsection{Minimum-Cell-Rate Criterion}

For the MCR criterion, the expected share for connection $p$ is

$$
E_{M C R}[g(p)]=\sum_{i=1}^{M} P\left(S_{k}=i\right) \cdot \frac{m_{p}}{m_{p}+(i-1) \cdot \bar{m}},
$$

where $\bar{m}$ is the mean MCR value of the connections encountered by connection $p$ at the bottleneck link and $m_{r} \equiv \frac{m_{p}}{\bar{m}}$. When $m_{r}=1$, the result is identical to the Max-Min criterion. In Fig.6, when $m_{r}>1$, a larger allocation compared to Max-Min Criterion will be given to connection $p$ because a higher priority has been assigned to $p$. When $m_{r}<1$, the bandwidth allocated to $p$ will be less than that of the Max-Min criterion.

\section{Numerical Results}

In the previous section, several assumptions have been made regarding the connections in the network. In this section, the PL and the MCR criteria are studied using a test configuration (Fig.7) which has been adopted by the ATM forum [5] for comparing the performance of different fairness criteria. There are six groups of virtual channels (VCs) namely $G_{i}\left(x_{i}\right)$, for $i=1, . .6$, with each of them consists of $x_{i}$ VCs. VCs in groups $G_{1}$ and $G_{2}$ are 3-link connections and VCs in other groups are 1-link connections. Bandwidth allocation to each $\mathrm{VC}$ is calculated using (4) to (7), and is shown in Table 1.

In Table 2, the average bandwidth allocation for the MaxMin criterion is calculated from Table 1. The beat down 
phenomenon can be observed beacuse the 1-link VCs is three times larger that of 3-link VCs.

For the PL criterion, the parameters $n$ and $K$ respectively represent the mean and maximum number of links traversed by the connections while $M$ denotes the maximum number of connections sharing a link. Hence, for this test configuration, $n=1.6, K=3$ and $M=9$. As shown in Table 3 , with $B=0$, the PL criterion is equal to the Max-Min criterion. With $B=3.35$, which is the optimum value of $B$ from (14), the average bandwidth allocation for the 3-link and 1-link VCs are approximately equal. With $B=10$, the 3-link VCs are favored more than the 1-link VCs and hence have a larger average bandwidth allocation.

With the MCR criterion, the MCR values of the 3-link VCs, $m_{3}$, are set to $0.4,1$ and 4 while that of the 1-link VCs, $m_{1}$, are set to 1 . As shown in Table 4 , the average bandwidth allocated for the 3-link VCs becomes larger when $m_{3}$ increases. This gain of average bandwidth is obtained at the expense of the 1-link connections. Therefore, the 3-link VCs can be given different priorities by simply assigning different values of MCR so that prioritized ABR service can be achieved.

\section{Conclusions}

The formulation of the GWFC allows the design of different ABR flow control algorithms with different performance objectives in mind. The Max-Min criterion, the PL criterion and the MCR criterion are the special cases under the GWFC.

Results show that the PL criterion is able to compensate for the beat down problem and the MCR criterion allows QoS-based prioritized ABR service to be realized.

\section{References}

[1] Dimitri Bertsekas and Robert Gallager, "Data Network", Prentice Hall, 1992, 2nd Edition.

[2] Jeffrey M. Jaffe, "Bottleneck flow control", IEEE Trans. on Comm. Vol. Com-29, No.7, July 1981.

[3] Kai-Yeung Siu and Hong-Yi Tzeng, "Adaptive proportional rate control for ABR service in atm netowrk", In Proc. Phoenix ICC'95, pages 529-35, March 1995.

[4] Flavio Bonomi and Kerry W. Fendick, "The rate-based flow control framework for the available bit rate atm service", IEEE Trans. on Network, April 1995.

[5] R. Simcoe. "Test configurations for fairness and other tests", ATM forum/94-0557, July 18, 1994.

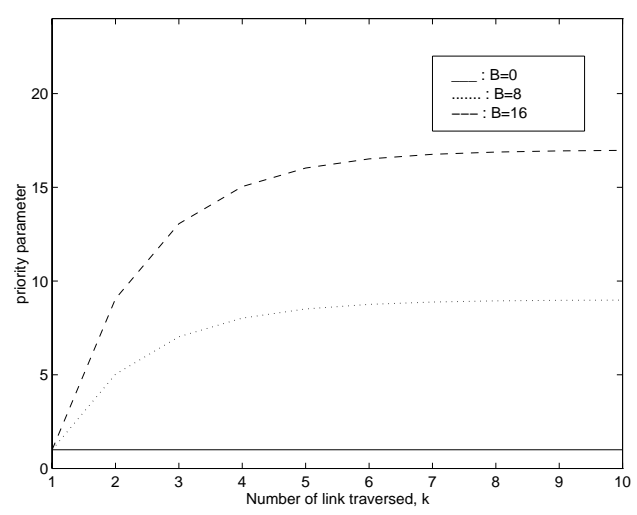

Figure 1. PL criterion : Weight profiles of a connection traversing $\mathbf{k}$ links

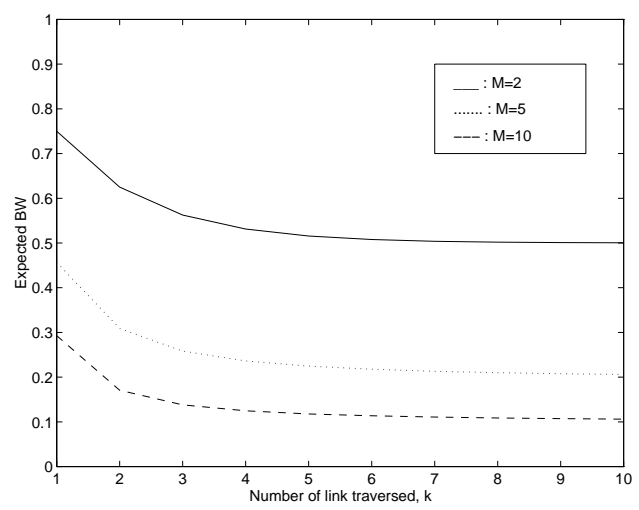

Figure 2. Max-Min criterion : Study of the 'Beat down problem'

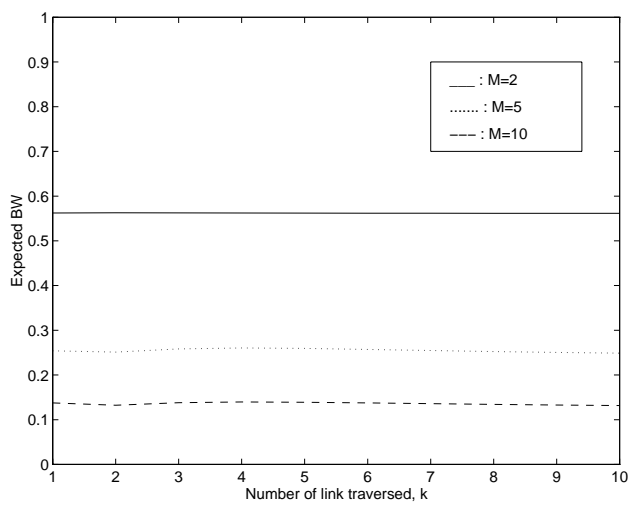

Figure 3. PL criterion : Study of the 'Beat down problem' with $n=3, B=8$ 


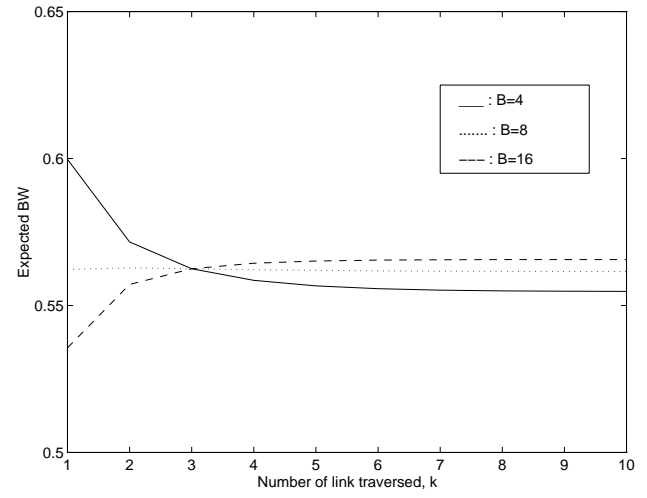

Figure 4. PL criterion : Effect of $B$ with $n=$ $3, M=2$

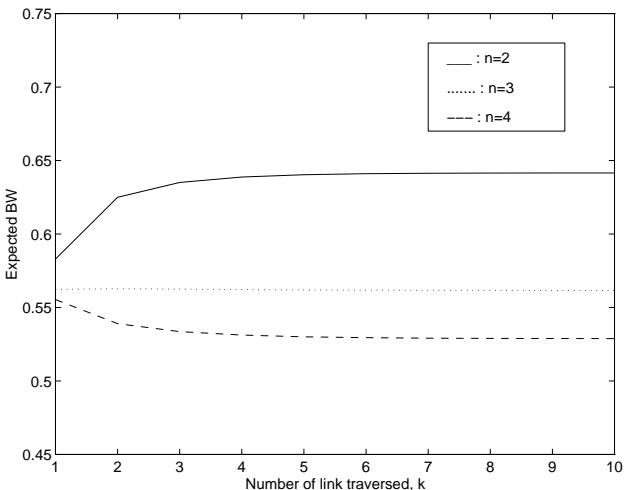

Figure 5. PL criterion : Effect of $n$ with $B=$ $8, M=2$

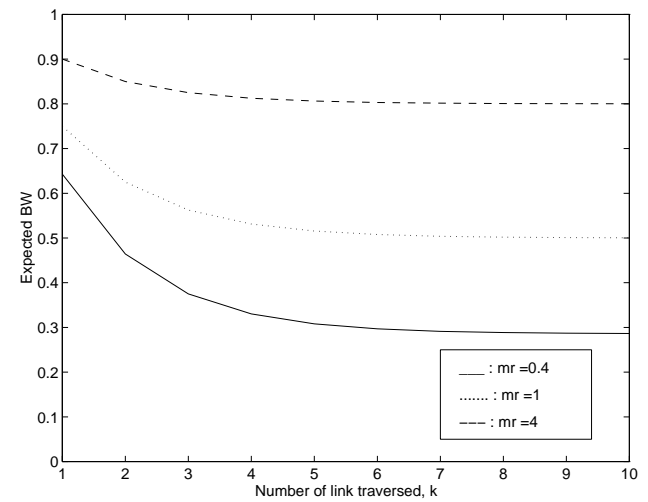

Figure 6. MCR criterion : Study of giving higher priority to a connection with $m_{r}=0.4,1$ and 4

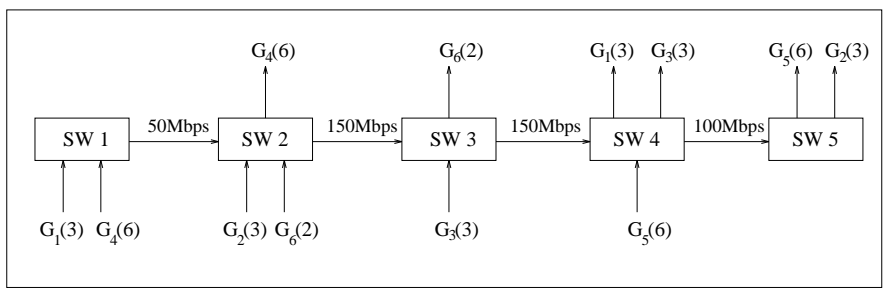

Figure 7. Test configuration

\begin{tabular}{|l|l|l|l|l|l|l|}
\hline Criteria & $G_{1}$ & $G_{2}$ & $G_{3}$ & $G_{4}$ & $G_{5}$ & $G_{6}$ \\
\hline Max-Min & 5.6 & 11.1 & 33.4 & 5.6 & 11.1 & 50.0 \\
\hline PL $(B=3.35)$ & 10.6 & 21.3 & 18.1 & 3.0 & 6.0 & 27.2 \\
\hline PL $(B=10)$ & 13.9 & 27.8 & 8.3 & 1.4 & 2.8 & 12.5 \\
\hline MCR $\left(m_{3}=0.4\right)$ & 3.3 & 6.7 & 40.0 & 6.7 & 13.3 & 60.0 \\
\hline MCR $\left(m_{3}=4\right)$ & 11.9 & 23.8 & 14.3 & 2.4 & 4.7 & 21.4 \\
\hline
\end{tabular}

Table 1. Bandwidth allocation for each VC in group $G_{i}, i=1, . .6$ (Mbps)

\begin{tabular}{|c|c|}
\hline $3-\operatorname{link}\left(G_{1}, G_{2}\right)$ & $1-\operatorname{link}\left(G_{3}, G_{4}, G_{5}, G_{6}\right)$ \\
\hline$\frac{5.6+11.1}{2}=8.4$ & $\frac{33.4+5.6+11.1+50}{4}=25.0$ \\
\hline
\end{tabular}

Table 2. Max-Min criterion : Average bandwidth allocation for the 3-link $\left(G_{1}, G_{2}\right)$ and the 1-link $\left(G_{3}, G_{4}, G_{5}, G_{6}\right)$ connections (Mbps)

\begin{tabular}{|c|c|c|}
\hline$B$ & $3-\operatorname{link}\left(G_{1}, G_{2}\right)$ & $1-\operatorname{link}\left(G_{3}, G_{4}, G_{5}, G_{6}\right)$ \\
\hline 0 & $\frac{5.6+11.1}{2}=8.4$ & $\frac{33.4+5.6+11.1+50}{4}=25.0$ \\
\hline 3.35 & $\frac{10.6+21.3}{2}=16.0$ & $\frac{18.1+3.0+6.0+27.2}{4}=13.6$ \\
\hline 10 & $\frac{13.9+27.8}{2}=20.9$ & $\frac{8.3+1.4+2.8+12.5}{4}=6.3$ \\
\hline
\end{tabular}

Table 3. PL criterion : Average bandwidth allocation for the 3-link $\left(G_{1}, G_{2}\right)$ and the 1-link $\left(G_{3}, G_{4}, G_{5}, G_{6}\right)$ connections (Mbps)

\begin{tabular}{|c|c|c|}
\hline$m_{3}$ & $3-\operatorname{link}\left(G_{1}, G_{2}\right)$ & $1-\operatorname{link}\left(G_{3}, G_{4}, G_{5}, G_{6}\right)$ \\
\hline 0.4 & $\frac{3.3+6.7}{2}=5.0$ & $\frac{40.0+6.7+13.3+60.0}{4}=30.0$ \\
\hline 1 & $\frac{5.6+11.1}{2}=8.4$ & $\frac{33.4+5.6+11.1+50.0}{4}=25.0$ \\
\hline 4 & $\frac{11.9+23.8}{2}=17.9$ & $\frac{14.3+2.4+4.7+21.4}{4}=10.7$ \\
\hline
\end{tabular}

Table 4. MCR criterion : Average bandwidth allocation for the 3-link $\left(G_{1}, G_{2}\right)$ and 1-link $\left(G_{3}, G_{4}, G_{5}, G_{6}\right)$ connections (Mbps) 